\title{
An Intelligent Driver Assistance System (I-DAS) for Vehicle Safety Modelling using Ontology Approach
}

\author{
Saravanan Kannan, Arunkumar Thangavelu, RameshBabu Kalivaradhan \\ School of Computing Sciences and Engineering, VIT University, \\ Vellore - 632014, TN, India \\ saravanank@vit.ac.in, arunkumar.thangavelu@gmail.com,ramgetkar@gmail.com
}

\begin{abstract}
This paper proposes an ontology modelling approach for assisting vehicle drivers through safety warning messages during time critical situation. Intelligent Driver Assistance System (I-DAS) is a major component of InVANET[12], which focuses on generating the alert messages based on the context aware parameters such as driving situations, vehicle dynamics, driver activity and environment.

I-DAS manages the parameter representation, consistent update /maintenance in XML format while the interpretation of a critical situation is done using ontology modeling. Related safety technologies such as Adaptive Cruise Control, Collision Avoidance System, Lane Departure Warning System, Driver Drowsiness detection system, Parking Assistance System, which generate warnings and alerts to driver continuously, for assistance according to context which is integrated in Vehicle and Vehicle 2 Driver (V2D) communications by DVI(Driver Vehicle Interface) had been applied.

The simulation test bed developed using Java framework[21] to generate safety alerts in various driving situations shows the usefulness of this approach. The response time graph for the simulation of context I$D A S$ is depicted and analysed. The effective performance of the driving scenarios in various modes like day and night for single, 2-way and 4-way road scenario for the best, worst and average cases of simulation had been studied. The system works in VANET scenario, which needs to be adaptive for environment changes and to vary according to the context. The presented approach shows the simulation that can be implemented to all vehicles in real time scenario with promising results.
\end{abstract}

\section{Keywords}

Context Awareness, Ontology Modeling, Driver Vehicle Interface(DVI), Driver Assistance System (DAS)

\section{Introduction}

Based on statistics[20][22], which reveals that road accidents on an average cause 1.2 million deaths and 50 million injuries around the world each year. However, these vehicles are prone to accidents due to issues as traffic congestion and rash driving, lane change over [12]. The context aware driver assistance system I-DAS aim to assisting driver according to the change $n$ environmental situation and respond to the context. Context-aware computing plays the major role of "performing the task" on behalf of the user depending on the situation i.e., interact with the environment in an autonomic manner and provides the human assistance which enables the researchers involved to developing context-aware applications in areas of distributed computing and HCI (Human-Computer Interaction). Hence, I-Driver Assistance System should be able to sense, analyze, predict and react to the road environment, which are the key features of contextawareness. Many technologies have been developed in the past decade, such as intelligent transportation systems (ITS) (Daniele Bagni et al [4]), the advanced driver assistant system (ADAS) [9]. The study on these works show that the information about the road environment, obstacles surrounding, environment within the vehicle, often termed contexts, is gathered. It is also to be noted that these research works lack in complex reasoning. These drawbacks limit the vehicles ability of assisting the driving task efficient and safe. The research on I-DAS focuses 
on building up of a context-aware driver assistance system using the existing technologies. The remaining sections of this paper is organized as follows. Section 2 introduces the related work on Intelligent Driver Assistance System, while Section 3 proposes an Ontology Modelling according to the context in comprehensive definition and classification of concepts of DAS and their control systems. The proposed Architecture is discussed in Section 4.The modelling and simulation in the software platform using JCreator[22] presented in Section 5. The conclusion and future work is discussed in Section 6.

\section{Need for DAS (Driver Assistance System) and Related Work}

Road situation analysis for driver assistance and safety warning is an interdisciplinary endeavour involving many research fields, for instance in computer science, automobile engineering, cognitive science, and psychology.

Several middleware ontology models are developed satisfying these criteria but all these mainly focused on navigation systems, infotainment applications and only few projects focused on safety related issues like MYCAREVENT [26] discussed on car to infrastructure and no adaptive middleware ontology support for vehicle to vehicle is focused. The VMTL project [25] is a web service based middleware for mobile vehicular applications focusing on navigation information that gives continuous location observation and tracking for tour data but not concentrating on safety issues. RT-Stream [23] is a space-elastic model proposed for realtime communications which mainly concentrates on medium access control and routing protocols. In this real-time model, the representation of data, its interoperability issues and reasoning about the situation were not discussed. In Context-Aware Rate Selection project CARS [24], an algorithm concentrating on packet transmission to achieve fast data rate adaptation in VANET is proposed. No middleware issues have been discussed in any of the above model. In the prototype Context-Aware Information Provision Services [27], notification of traffic problems and warning messages of congestion forecasts transmitted over Peer to Peer JXTA overlay network contains the message source, event type and event payload. This is again a publish subscribe type middleware where group servers and storage servers are necessary to invoke the functionality.

The multiplatform Open Source Gateway Initiative, OSGi based architecture for developing road vehicle services [28] uses Java Service Interfaces through JavaComm, Java Media Framework and Java Speech. This project mainly focuses on Navigation, Infotainment but not about safety parameters. The scene representation and the related technologies for the Driver assistance system are studied below.

\subsection{Scene Representation of Driver Assistance System}

We classify existing framework simulation that analyze obstacles in a traffic scene using control systems like Adaptive Cruise Control, Collision Avoidance System, Lane Departure Warning System, Driver Drowsiness detection system, Parking Assistance System [18].The current situation analysis framework, which attempts to provide the vehicle and the driver with the obstacles' information in the current time by fusing different sensors [4], LIDAR and radar as XML inputs. Real-time safety analysis in a traffic environment involving driver, vehicle itself, and their interaction is a challenge for perception, modelling, decision, and control. Several safety analysis systems have been proposed to address different aspects in road situation analysis. In addition, accidents are caused due to inappropriate speed, absence of traffic signs, wrong turning, and pedestrian mistake. The radar, lidar and camera XML inputs are fused and processed for detection of vehicles, objects in environment. The project aims at avoiding collisions between pedestrians and vehicles. This paper proposes an both integrated for current and future safety situation analysis framework as possible, where we model not only the sensing phase but also the system response phase. Combining the lane structure with obstacle 
tracks, we can model the traffic environment and assess road situation at both the current and near-future time.

\subsection{Technologies used in Driver Assistance System}

The following technologies are studied for simulation of the Intelligent Driver Assistance System for safety warning.

\subsubsection{Adaptive Cruise Control (ACC)}

ACC has been under development since the Prometheus (Programme for European Transport with Highest Efficiency and Unprecedented Safety) programme began in 1986. Today's ACC relies on radar or laser technology to track a vehicle ahead and maintain a safe gap. It lets the vehicle hold a speed but adjusts to changing traffic conditions with automatic braking and acceleration [6][8].ACC reduces the number of sudden accelerations and decelerations, enables speed synchronization among vehicles, and encourages smooth lane change behaviors, and reduce accident possibility[17].When situation is out of hand the vehicle is decelerated and stopped in worst conditions for the safety of the driver.

\subsubsection{Lane Departure Warning}

Lane departure warning alerts the driver when the car begins to leave its lane without obvious input from the driver (for instance, when the driver is distracted or is very tired)[10]. A video camera in the rear view mirror allows the electronics to track the lane markings on the road ahead. Using this video image the software determines the car's position in the lane and then compares this position with additional inputs taken from the steering angle, brake and accelerator position sensors - and whether or not the indicators are in use[10][1]. If the car begins to drift off track for no apparent reason, the driver is alerted by an audio or haptic warning such as a vibrating the seat and match all the different available information and then decide to display on HMI.

\subsubsection{Parking assistance}

Citroën C4 Picasso's is the world's first car to feature integrated parking space management. The parking space measurement system [1] [12] is based on the supplier's parking assistant, which helps drivers avoid shunting other cars and obstacles while parking. While driving at a speed of up to $20 \mathrm{~km} / \mathrm{h}$ - the sensor then gauges the space. The assistant indicates to the driver via the central display whether the gap is big enough for the car, a tight squeeze, or simply too small. The same visual and acoustic signals that feature in the parking assistant then warn the driver of any obstacles.

\subsubsection{Collision Avoidance System}

The introduction of collision warning/avoidance systems allows the driver to take appropriate corrective actions in order to mitigate, or completely avoid the collision event [11]. Some of the dangers that sensors can pick up include how close the vehicle is to other vehicles surrounding it, how much its speed needs to be reduced while going around a curve, and how close the vehicle is going off the road. The system uses sensors that send and receive signals from other vehicles; obstacles in the road [15]. A situation that provides a good example of how the system works is when a driver is about to change lanes, and when the vehicle is in blind spot. The sensors will detect that vehicle and inform the driver, preventing him from potentially getting into a serious accident. 


\subsubsection{Driver drowsiness detection}

Drowsy driving', sleep-related vehicle accidents (SRVA's) are a common type of road crash. In case of hypo vigilance, this system provides an adequate warning to the driver, with various levels of warnings, according to the estimated driver's hypo vigilance state and also to the estimated level of traffic risk. The Driver Warning System (DWS) uses different modalities acoustic, visual and haptic output signals to warn the driver against his drowsiness which prevents from accidents [5].

\subsubsection{Automotive Night vision}

There are many disciplines and components involved to make a Night Vision system into an interesting assistance system from a technical point of view [7]. Systems which make pedestrian recognition possible in Night Vision systems will certainly be an additional advantage. It increases driver's perception and seeing distance in darkness or poor weather beyond the reach of the vehicle's headlights.

\section{I-DAS Proposed Architecture}

The overview of our proposed architecture is shown in figure 1 . The inputs are taken from the driver, vehicle, and external environment sensors and given to the system. The inputs are collected and fused according to the context. The acquired input data is assimilated according to their respective contexts and sent to Context Aware Reasoning. The contextual inputs are compared with the particular control subsystems context representation and domain knowledge to invoke safety warnings before event takes place for safer action to be taken within that period of time through DVI and store the action taken place in database. The architectural diagram of the Context Aware DAS for safety warning is given below in figure 1.

Context Acquisition \& fusion works on taking inputs [12] (refer work on InVANET) from sensors, radars, lidar for various context aware situations as an XML document and parses the XML file using SAX parser. The related XML elements for a particular context generate alerts or warnings. The parsed XML elements are given to the scenario and displayed as a car processing details in car simulation.

Context Reasoning engine is required to respond to the context aware situations invoking the control subsystems ( Adaptive Cruise Control (ACC), Lane Departure Warning (LDW), Parking Assistance, Automotive Night Visions System, Collision Avoidance System and Driver Drowsy detection System) defining various rules and logical conditions to generate alerts and warnings like to keep safe distance between vehicles, reduce speed, passengers passing from front detected, parking of vehicles is successful, vehicle changing from one lane to another, vibrating the seat of drowsy driver etc.

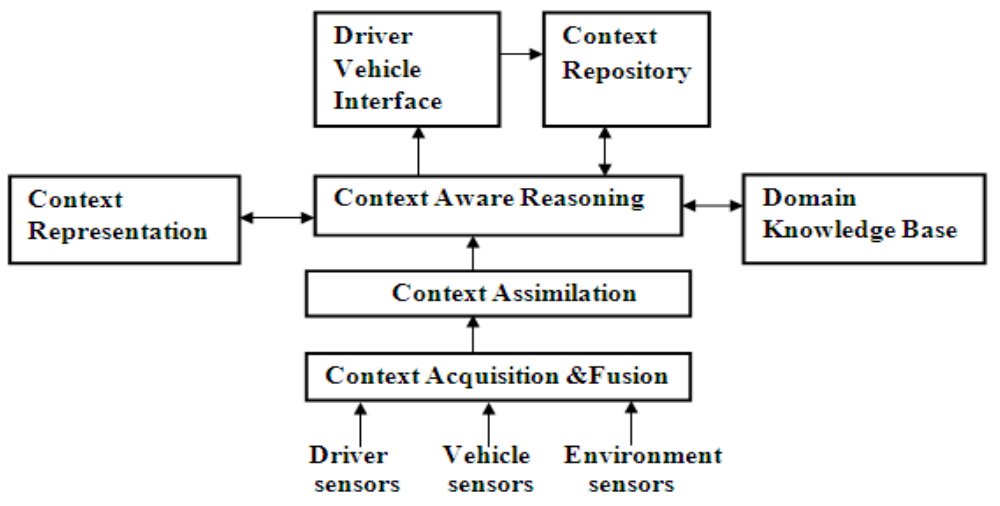

Figure 1: Architecture of Intelligent Driver Assistance System 
Driver Vehicle Interface gives the GUI of road scenario for single, 2-way, and 4-way and generates alerts and warnings to driver invoking the control subsystems in day mode and night mode according to the Context .The line chart graph is shown in GUI depicting the performance of the system.

Context Database stores some of the pre history data about the alerts, warnings, and messages of which expected situations to happen and respond accordingly. Suppose, new situations occurs we define some predefined rules to react according to the context.

The following steps are followed for the detailed DAS Architecture proposed in Figure 2.

Step 1: Collect sensor data from the lidar, radar from sensor environment as xml file.

Step 2: Parse input and feed to the Context Acquisition Fusion.

Step3\&4: Look Up the Context Database for such situation has occurred previously and Retrieve relevant data according to the context and Return to Context Acquisition Fusion.

Step5: If the data relevant to the particular sensors then assimilate the Context and input to Context Service Engine. Goto step 9.

Step6\&7: If it is newly sensed data, learn it and Update newly learned data in the database.

Step8: If it is learned that it is new Action/Service it is sent to Action/service database.

Step9: Context Service Engine query's a request to Service/Action Database.

Step10: Response from Action/service database is given to Context Service Engine.

Step 11: Context Engine perform the action and inputs to API/ Agent.

Step 12: Agent/API generates the Alerts/warning signals to the Human Vehicle Interface.

Step 13: The action performed is stored in the log database.

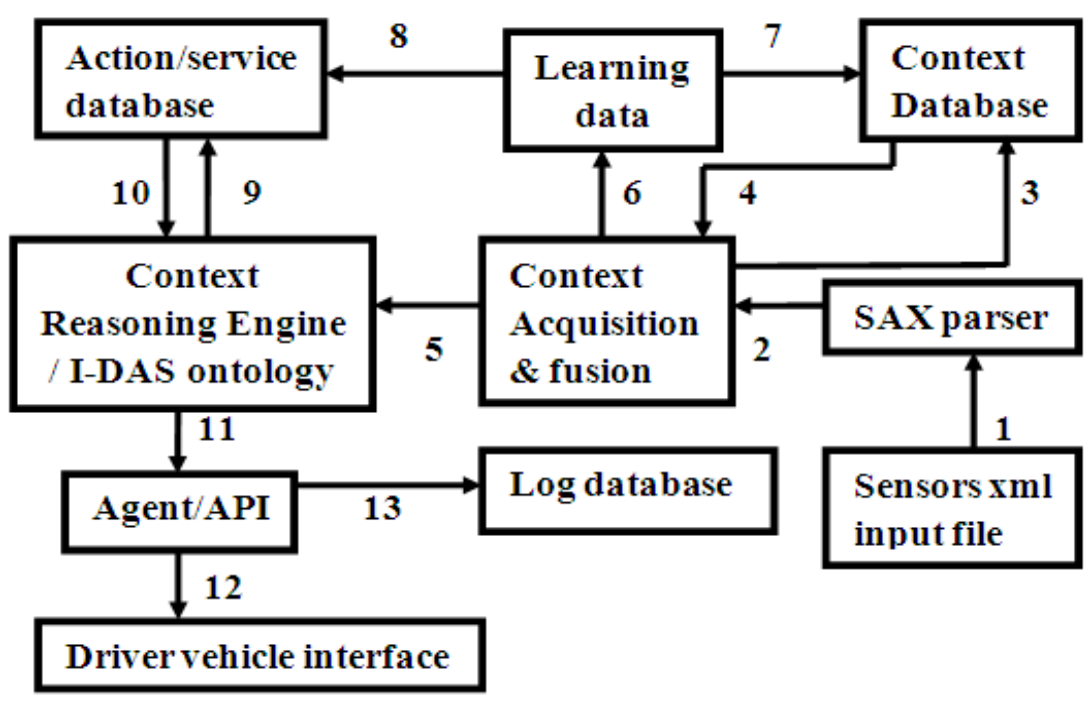

Figure 2: Functionality of I-DAS 


\section{Ontology Modelling}

The experimental approach on key based value models, object oriented models, logical models and Graphical models which are used in Artificial Intelligence, Situation Theory, Interactive Environment are proven to be inefficient, difficult to capture complex contextual relationship \& constraints, poor performance in real time, error-prone, incomplete, ambiguous and have less applicability issues[3]. As Ontology Modelling [13][14][15] has wide range of uses in psychological studies, ubiquitous computing, distributed / interoperable systems and intelligent systems [14], OWL was finally chosen over other models to facilitate the common information representation and exchange process between collaborating vehicles for intelligent transport systems for reuse, interoperability, compatibility, knowledge sharing, logic inference, brokercentric, and agent based [2][3]. OWL-based context-model for representing information about objects and relationships relevant for the driving assistance task as developed. We start with design of ontology, utilizing the OWL-modeling tool "Protégé3.4" [19]. The scene description of Context Driver Assistance is modelled using Specification Description Language to map into logical simulation shown in Figure-3.

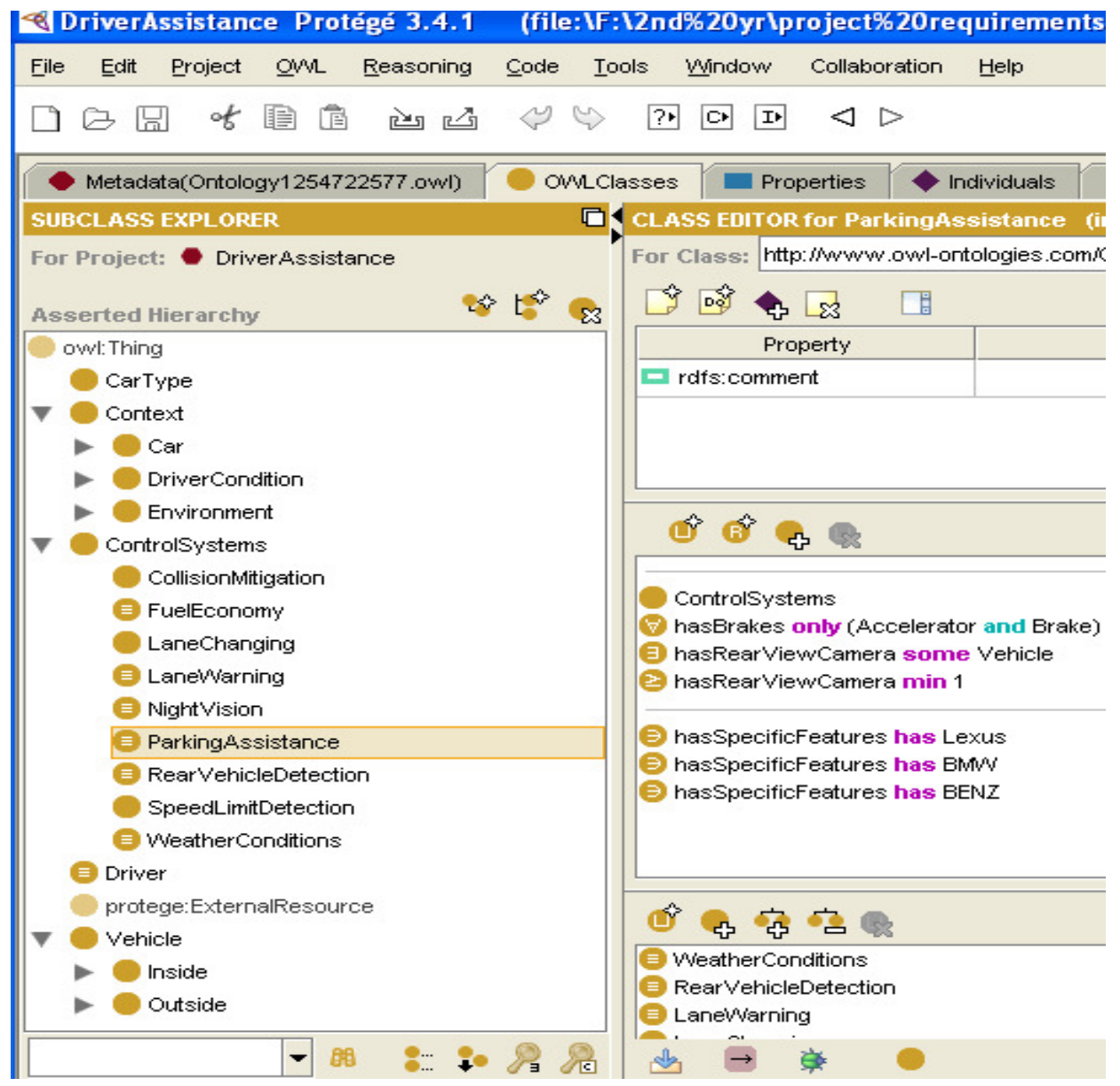

Figure-3: Ontology modelling of Intelligent Driver Assistance System

Traffic objects are included from four major context categories: driver, in-vehicle, traffic regulations and driving environment with respect to the vehicle [15].These objects are represented as classes (concrete representations of concepts), individuals (instances of classes) and properties (relationships between individuals).The consistency and taxonomy classification 
is checked using reasoner (pellet). After the reasoner has been invoked, computer super classsubclass relationships and inconsistent classes are displayed in the 'Classification Results' pane. If a class has been found to be inconsistent its icon will be circled in red.

The task of computing the inferred class hierarchy is also known as classifying the Ontology. Owlviz plug-in is used to design the class hierarchy and define relationships between them. Traffic objects have relationships to control systems like Adaptive Cruise Control, Collision Avoidance System, Lane Departure Warning System, Driver Drowsiness detection system, Parking Assistance System, while the Visualization plug-in for the Protégé is to show the hierarchy of classes and incrementally navigate in Owlviz (figure 4). Primitive classes that have no equivalent classes or definitions are shown in yellow. Defined classes that have at least one equivalent class are shown in orange. Edges between classes are shown in light grey, unless they join the selected class with another class and subclass edges are shown in purple. If a class has been reclassified, then its border and label will be shown in blue.

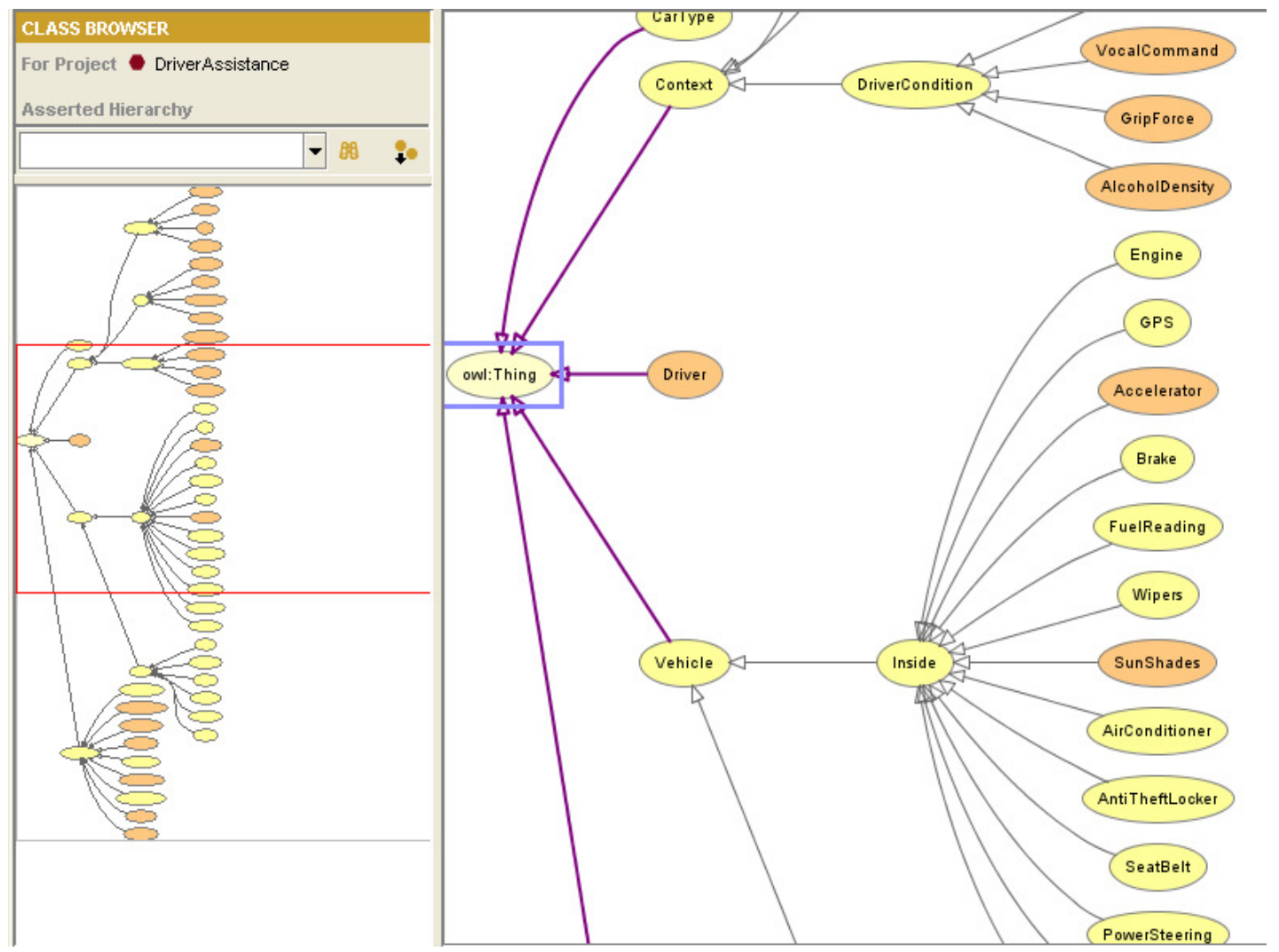

Figure-4: Hierarchy of I-DAS classes and relationship

\section{Simulation}

The modeling of context aware driver assistance system is worked on protégé OWL 3.4 , and the needed driving scenario descriptions of vehicle, environment and driver are modelled along with all the control systems like Adaptive Cruise Control, Collision Avoidance System, Lane Departure Warning System, Driver Drowsiness detection system, Parking Assistance System and automatic night vision system .This is sufficiently exhaustive that represents all relevant objects in a driving situation, as well as their relationships to environment and the vehicle together called DAS ontology. The model can be easily extended, without changing existing 
dependencies, using a step-wise refinement process exploiting OWL class hierarchies. Applications can enhance the class descriptions to a higher detail-level, any time, if needed. These description concepts that are developed in DAS ontology are control systems according to the contexts that occur in road scenario. This developed ontology is used for logical simulation of DAS. Next, the input sensor XML files are created and send to SAX parser to be parsed. The sensor.xml file is given below which contains the properties and elements of car length and breadth, initial speed, model, front and rear lidar coverage, left and right range radar , monitoring time ,lane width, parking length and breadth, humidity value, rain sensor value, camera visibility range, angle and image color.

\section{Sample input XML file i-das.xml}

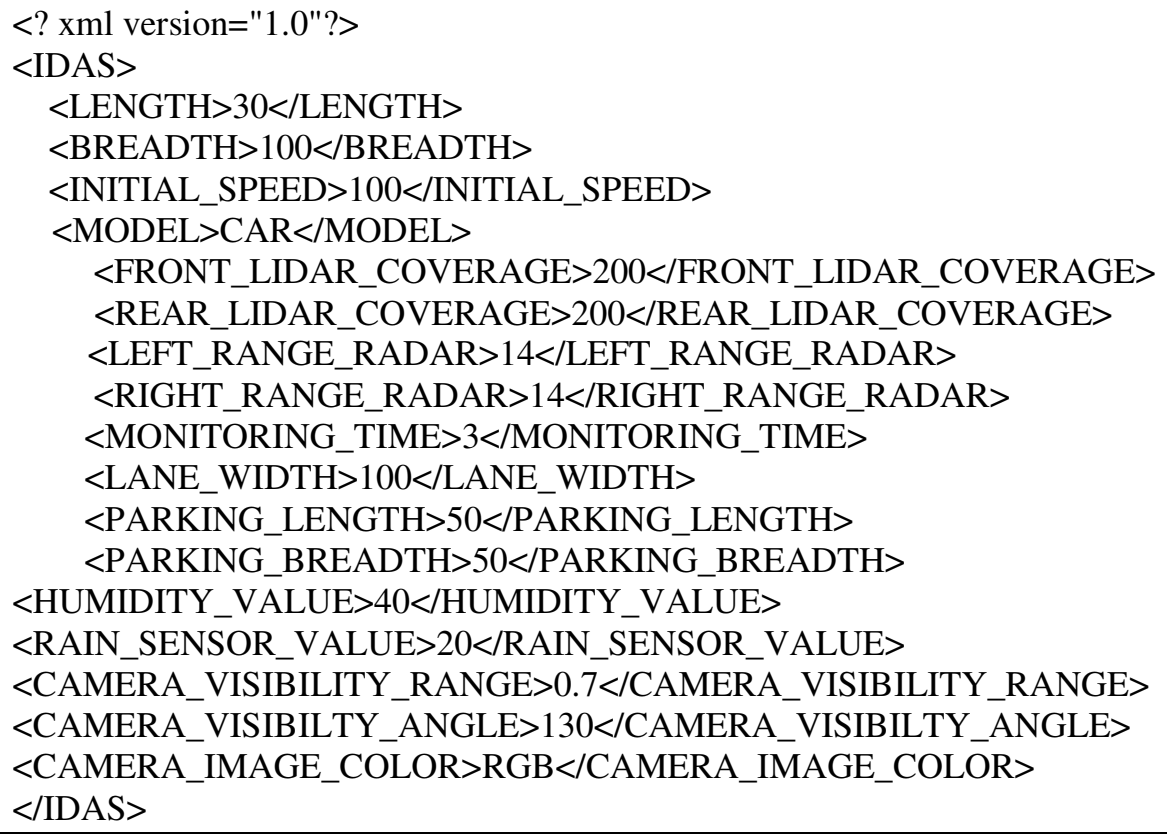

The parsed XML elements are given to the context acquisition and fused according to the contextual situation. These contextual elements are reasoned with context reasoning engine/DAS ontology i.e. the control systems to be invoked and necessary safety warnings are generated. The Simulation is done in the Java IDE JCreator implementing the road scenario in GUI and control systems Adaptive Cruise Control, Collision Avoidance System, Lane Departure Warning System, Driver Drowsiness detection system, Parking Assistance System, and Automatic night vision system for generating the alerts and warnings. These control systems are adaptive to the environment changes and behaves according to the context such as intelligent speed adaptation, detecting the obstacles and maintaining safe-distance, driver drowsiness detection, parking assistance and remote diagnostics of pedestrians, obstacles are shown for the day and night modes in single, double and four ways showing the actual scenario how the real system would response to the context in figures 5,6,7,8,9.

In figure 5 snapshot shows alert about obstacle (dustbin) and passenger on the road is messaged to driver where collision avoidance system is invoked. The sensor car processing details are shown in the GUI window of the same snapshot in figure 5. In next snapshot of control subsystem lane departure warning system is invoked alerting driver about the lane diversion from one lane to another is shown in the figure 6. The figure 7 snapshot shows the adaptive cruise control invoked in day mode alerting driver to reduce speed and maintain safe distance between vehicles and passengers. The Figure 8 is a snapshot that shows the driver 
drowsy detection system invoked when the driver is found sleepy and alerting him by vibrating the seat with beep sound. Figure 9 shows the Parking assistance system of the car.

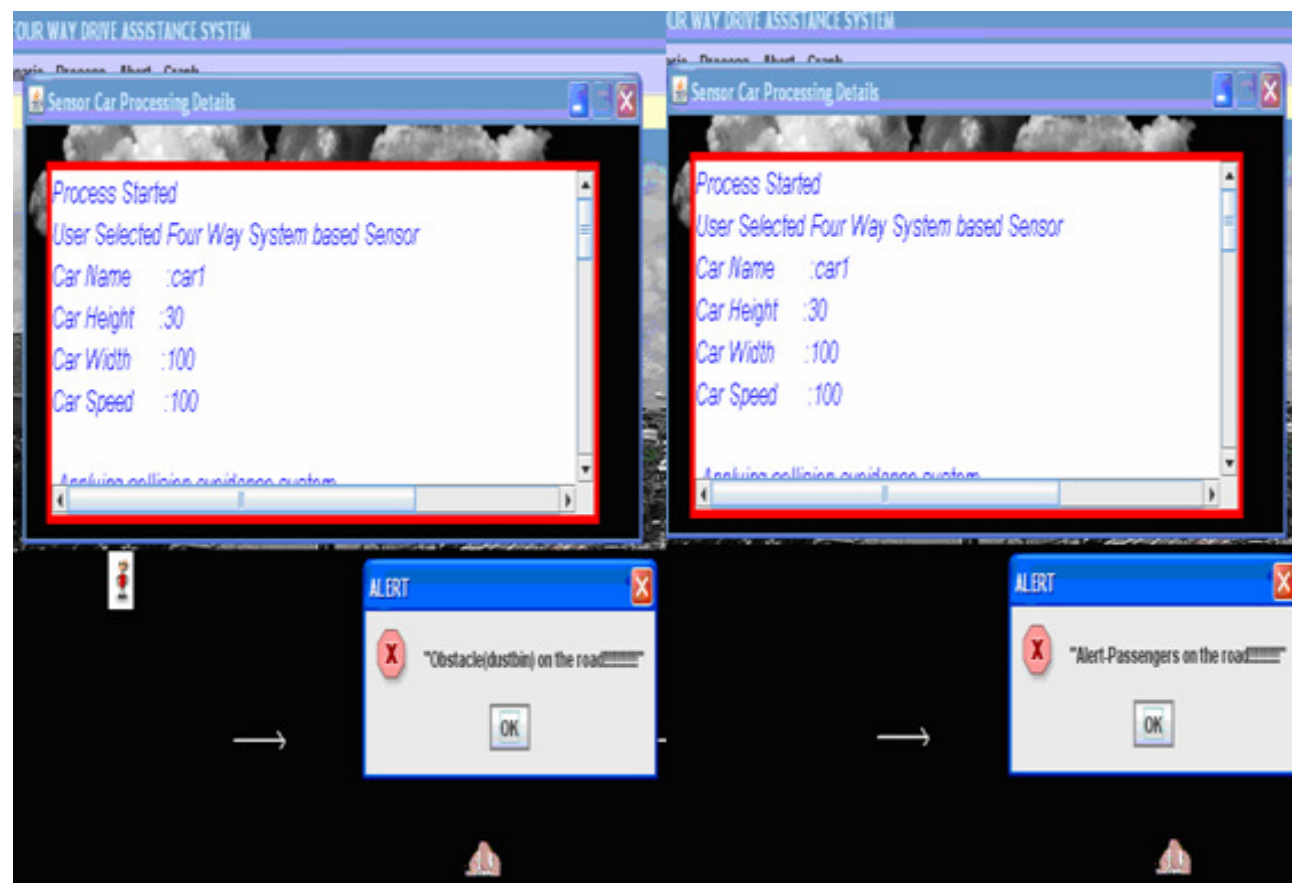

Figure5: Alert about passengers and obstacles on road using Collision avoidance system

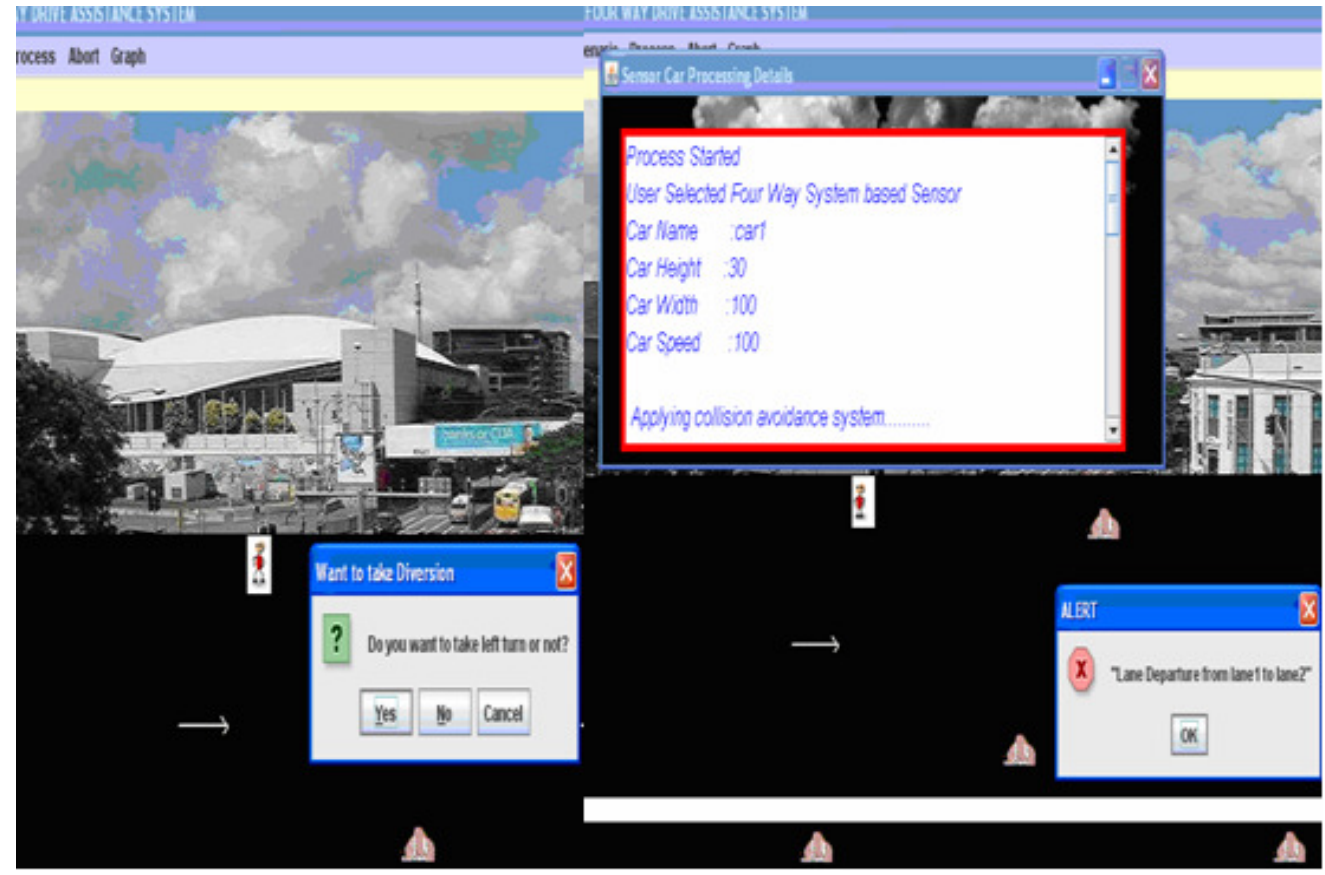

Figure 6: Alert about lane departure warning in day mode 
International Journal Of UbiComp (IJU), Vol.1, No.3, July 2010

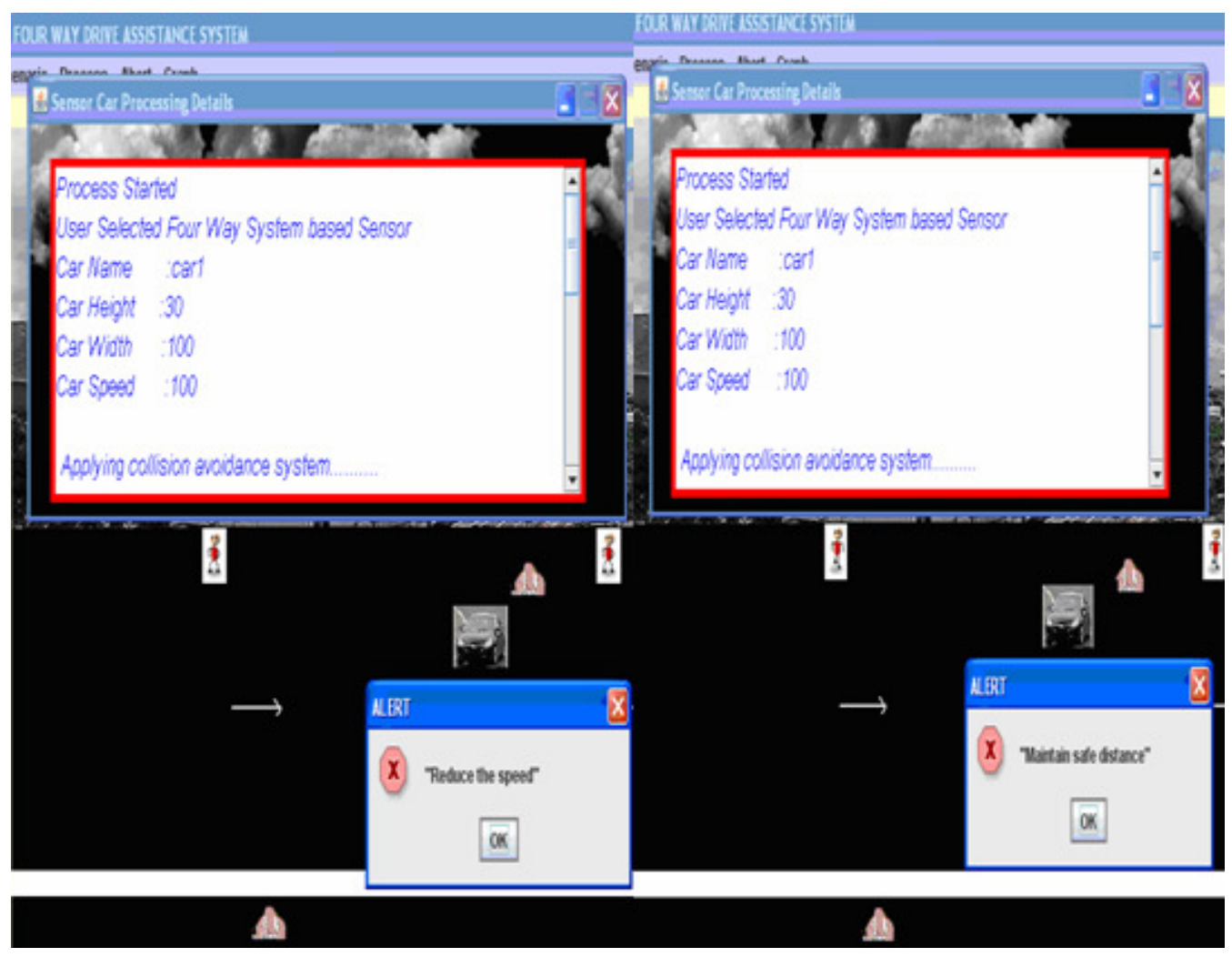

Figure 7: DAS in Day mode for Adaptive cruise Control

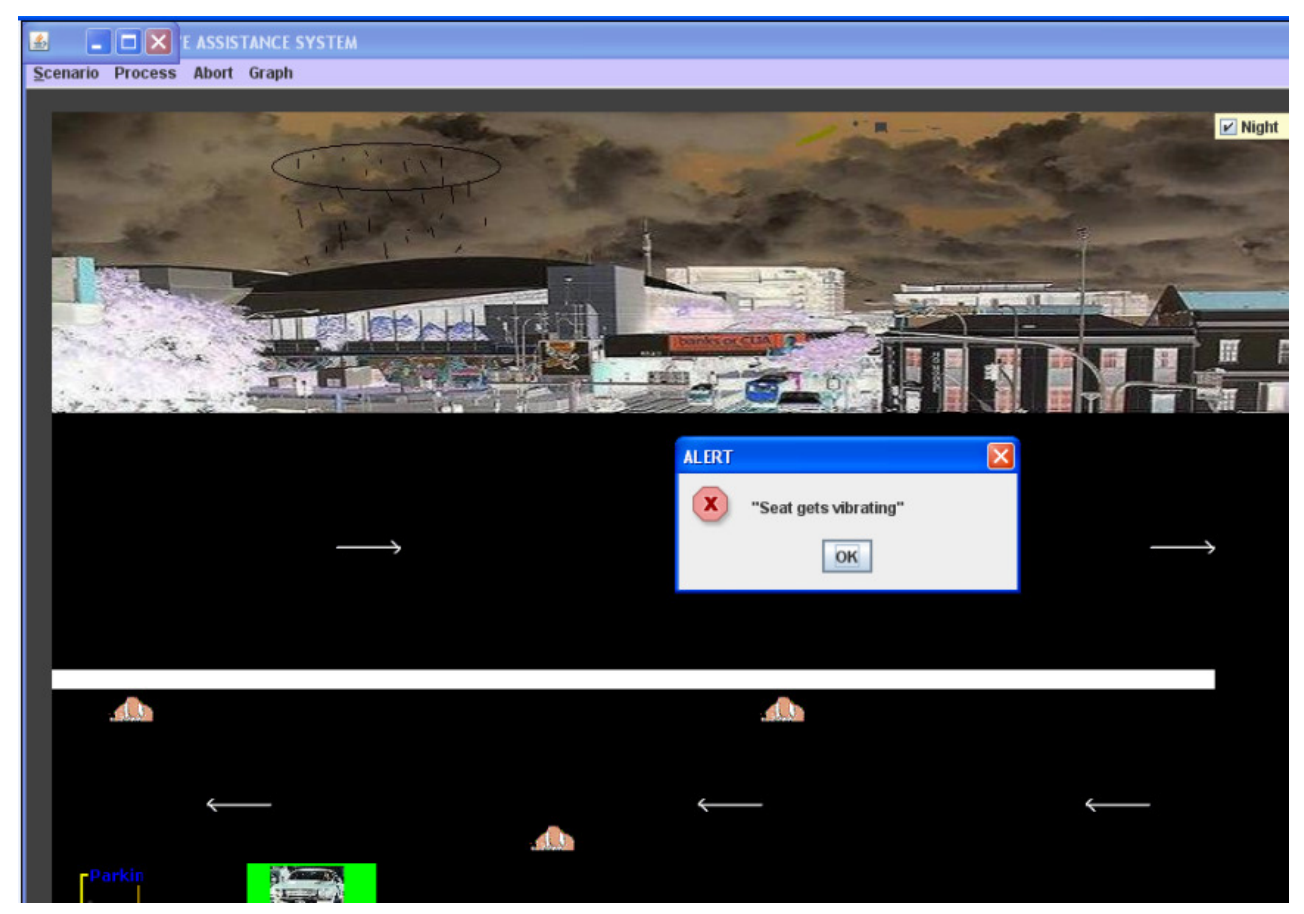

Figure 8: I-DAS invoking Driver drowsy detection system 


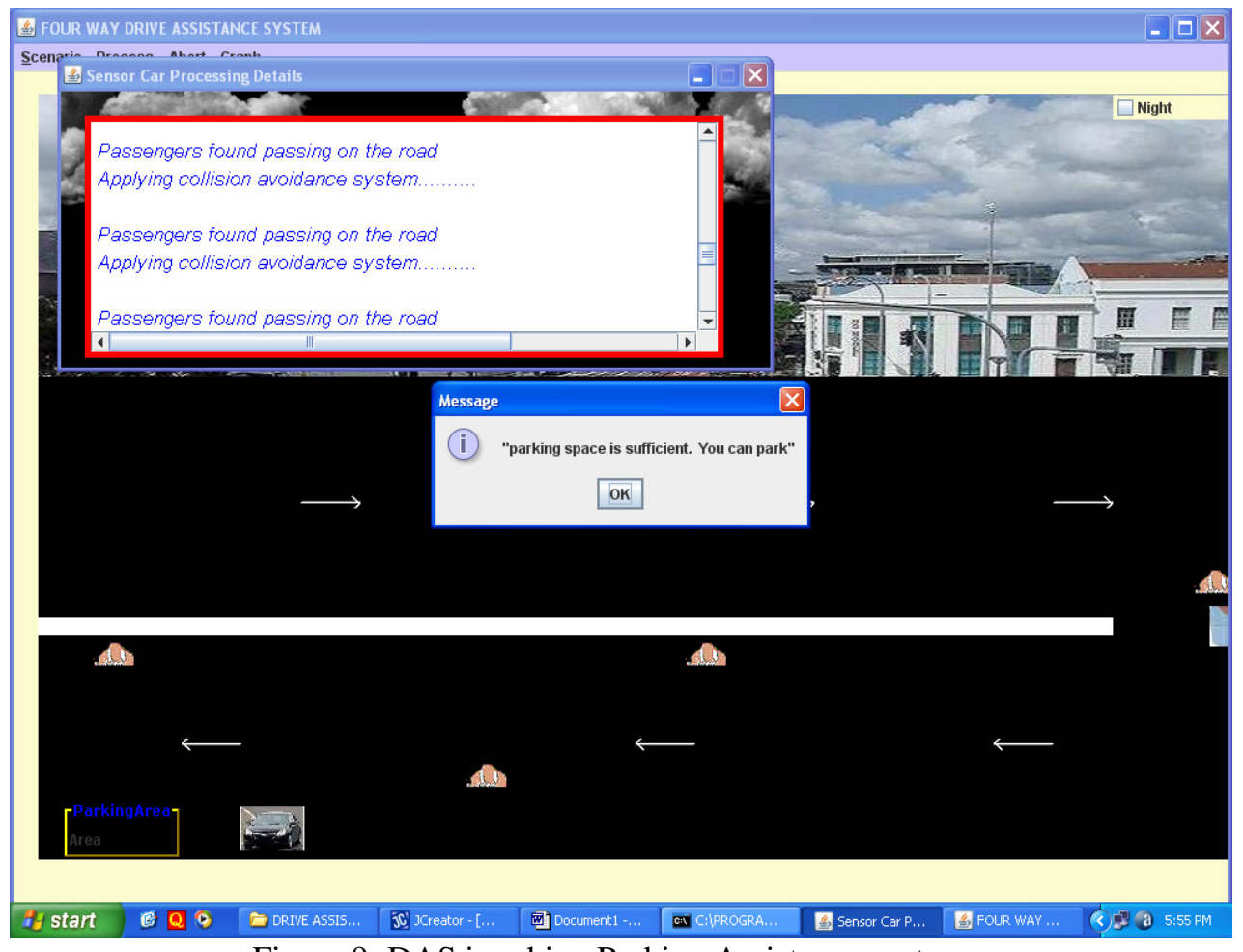

Figure 9: DAS invoking Parking Assistance system

The simulation of context Aware Driver Assistance System Safety warnings is depicted to show the response time graph.The $\mathrm{X}$-axis indicates the time at which the control system is invoked and on Y-axis indicates the time at which the warnings are generated. The response time is measured in milliseconds between the time of control system is invoked to the time the warning arises is shown in Figure 10,11,12. The red coloured notation shows single way response time, blue coloured line shows 2-way response time and green colored line shows 4-way response time.

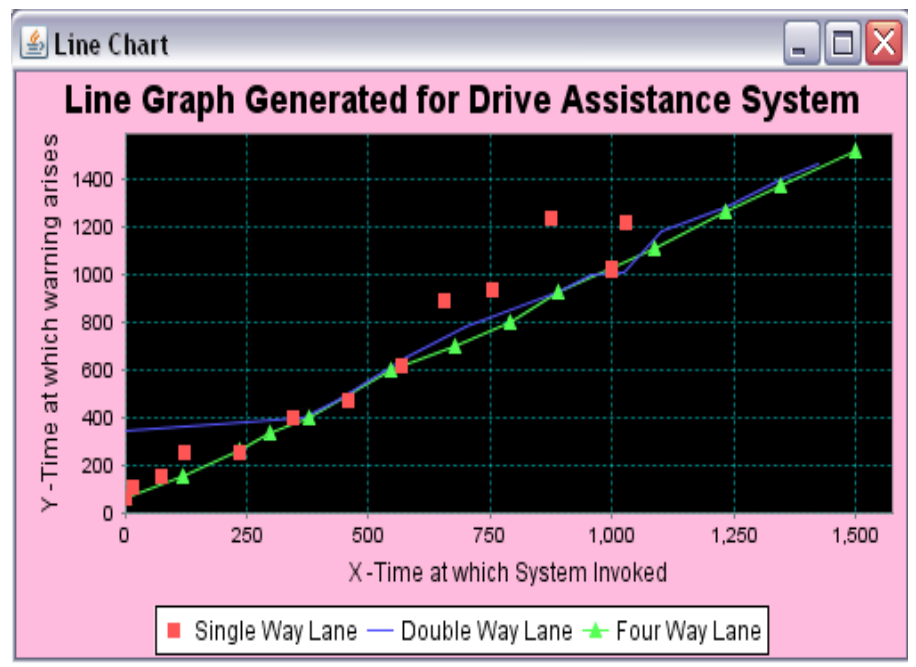

Figure 10: Best case scenario of DAS simulator response time graph 


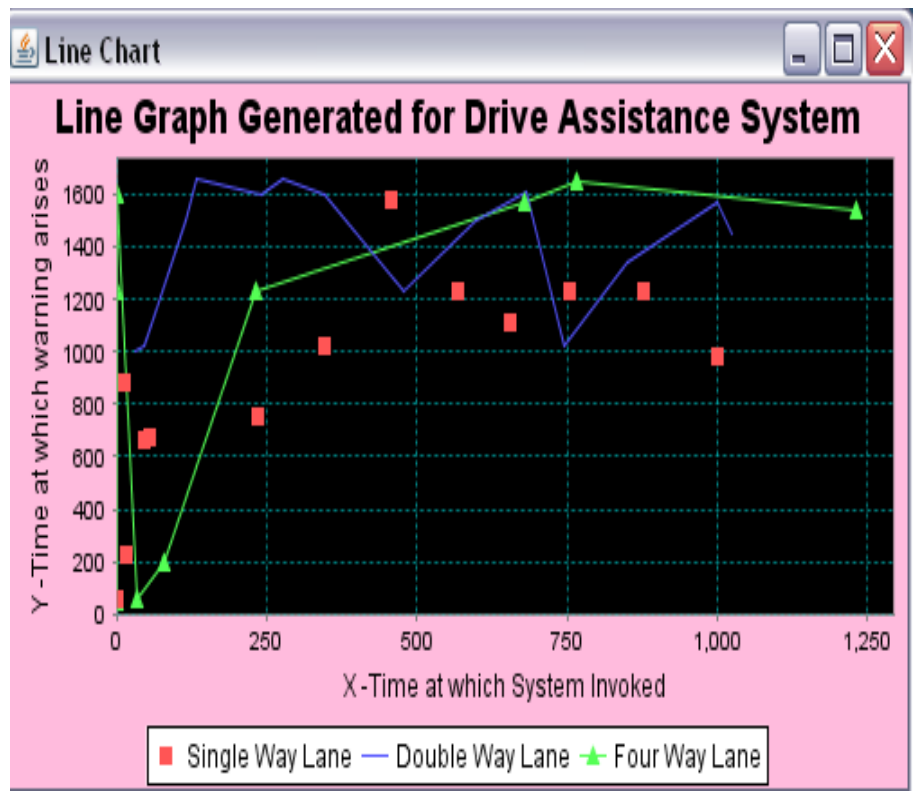

Figure 11: Worst case scenario of DAS simulator response time graph

The best case scenario is shown in the Figure 10. In this case, the graph for context aware DAS safety warnings generated for 1-way, 2-way, and 4-way is linear. This indicates that the response time of the warnings is highly immediate and spontaneous responding to the context by invoking the control subsystems efficiently. The worst case scenario is shown in the Figure 11. In this case, the graph for context aware DAS safety warnings generated for 1-way, 2-way, and 4-way has many peaks and ridges. This indicates that the response time of the warnings is not immediate and spontaneous with inefficient response to the context invoking the control subsystems with latency. The average case scenario is shown in figure 12. In this case the graph has constant response time for safety warnings generated for many of the contexts that invoke the control systems immediately and within constraint period of time. Thus the context aware DAS simulator would have a feasibility of implementing in real time.

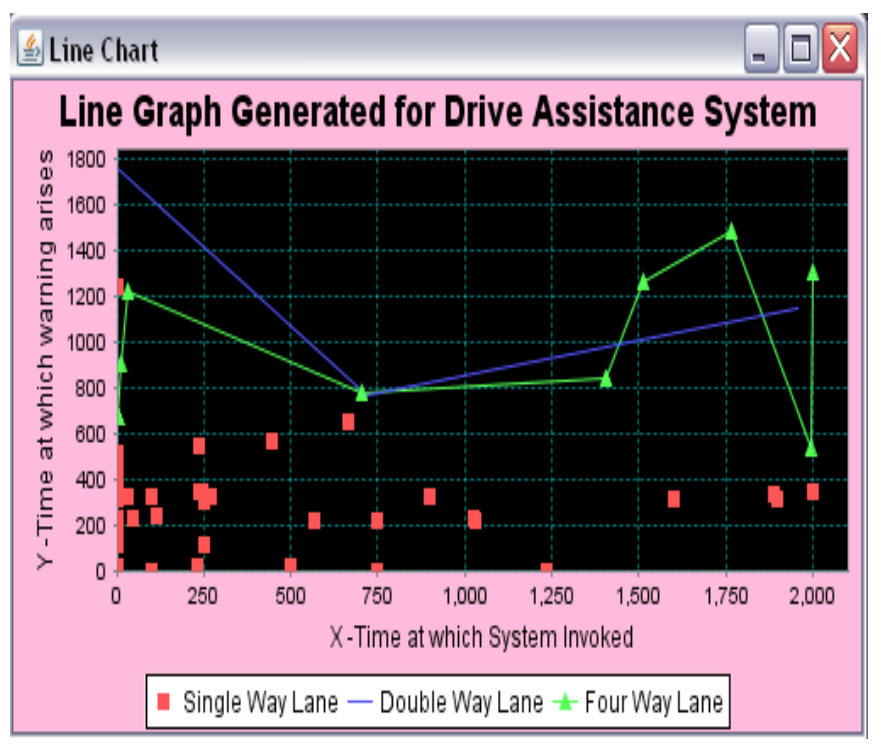

Figure 12: Average case scenario of DAS response time graph 


\section{Conclusion and Future Work}

In this paper, we study the issues and challenges of safety warning message exchange for the Context Aware Driver Assistance System. In general, driver assistance system demands a common domain understanding for scene representation to enable information exchange between vehicle and driver. We developed an ontology-based driving assistance context-model (I-DAS) which, explains a representation of variety of control systems that serve as common basis for domain understanding, decision-making and information sharing. We further discuss the integration of modelled scenarios within a logic programming environment in JCreator[22], using OWL's support for rule representation. The performance of the simulated context I-DAS safety warnings in best, worst and average case scenarios is discussed. The presented approach shows the simulation that can be implemented in real time scenario as DVI, which generates promising results and demonstrates the feasibility of combining the advantages of ontology with the reasoning power of logic-based languages.

Future work will be concerned with a more comprehensive extension of the overtaking knowledge base that is able to adapt its decisions to the individual needs of the driver and that takes the meta-information of traffic objects into account during the reasoning process.

\section{References}

[1] Akira Iihoshi, "Driver Assistance System (Lane Keep Assist System)", Presentation to WP-29 ITS Round Table Geneva, 2004.

[2] Bouquet, Petal, "Theories and uses of context in knowledge representation and reasoning", Journal of Pragmatics, vol no-335, pg 455-484, 2003.

[3] Bradley, "A multidisciplinary model of context to support context-aware computing", HumanComputer Interaction vol 20, pg 403-446, 2005.

[4] Daniele Bagni, Roberto Marzotto, Paul Zoratti, "Building Automotive Driver Assistance Algorithms with Xilinx FPGA platforms”, Xcell Journal Fourth quarter 2008.

[5] E.Bekiaris, S.Nikolaou, A.Mousadakou, "System for effective Assessment of driver vigilance and Warning according to traffic risk Estimation", National Center for Research and Technology, Hellas (CERTH) AWAKE Consortium August 2004.

[6] Hella KGaA, Hueck \& Co, "Electronics - Driver Assistance Systems", Technical Information, 2005.

[7] Hella KGaA, Hueck \& Co, “Light - ADILIS Night Vision System”, Technical Information, 2007.

[8] Huei Peng, "Evaluation of Driver Assistance Systems- A Human Centered Approach", supported by the U.S. Army TARDEC, NSF and the TRW Automotive,2006.

[9] Jie Sun, Zhao-hui Wu, Gang Pan, "Context-aware smart car: from model to prototype", Journal of Zhejiang University Science A,10(7):1049-1059, 2009.

[10] K.Henricksen, J.Indulska. "Software Engineering Framework for Context-Aware Pervasive Computing”, 2nd IEEE Conference on Pervasive Computing and Communications (PerCom), 2004.

[11] Peter Seiler, Bongsob Song, J.Karl Hedrick, "Development of a Collision Avoidance System", Society of Automotive Engineers 1998.

[12] Saravanan K, Arunkumar Thangavelu, Rameshbabu, "A Middleware Architectural framework for Vehicular Safety over VANET (InVANET)", NETCOM 2009, First International Conference on Networks \& Communications, pp.277-282, 2009.

[13] Simone Fuchs, Stefan Rass, Bernhard Lamprecht, Kyandoghere Kyamakya, “A Model for OntologyBased Scene Description for Context-Aware Driver Assistance Systems”,ACM SIGCHI, ICST Canada, 2008.

[14]Simone Fuchs, Stefan Rass, Kyandoghere Kyamak-ya, "Integration of Ontological Scene Representation and Logic-Based Reasoning for Context-Aware Driver Assistance Systems", Proceedings 
of the First International DisCoTec Workshop on Context-aware Adaptation Mechanisms for Pervasive and Ubiquitous Services (CAMPUS 2008),2008.

[15]T.A.Lasky,K.S. YEN,B.Ravani, "The advanced snowplow Driver Assistance system" supported by Caltrans New technology and new Program through (AHMCT) program at UC-Devis under IA65X875TO-96-9,2009.

[16]Tom Airaksinen, Hedvig Aminoff, Erik Byström, Gustav Eimar, Iracema Mata,David Schmidt, "Automatic Parallel Parking Assistance System User Interface Design - Easier Said Than Done?”,Technical Description, 2004.

[17]W.S. Lee, D.H. Sung, J.Y. Lee, Y.S. Kim , J.H. Cho,"Driving Simulation for Evaluation of Driver Assistance Systems and Driving Management Systems", sponsored by the Korea Transportation Institute under the national project, 'Development of National Traffic Core Technology',2007.

[18]Xuetao Zhang, Junjie Qin, Huub van de Wetering, "Interactive Road Situation Analysis for Driver Assistance and Safety Warning Systems: Framework and Algorithms", IEEE Transactions On Intelligent Transportation Systems, Vol. 8, No. 1, March 2007.

[19] http://protege.stanford.edu/ Protégé Software

[20] http://safety.transportation.org/htmlguides/RORcrashes/descriptionofstrat

[21] http://www.jcreator.com/

[22] Bray, J. Memo on "Skid Accident Reduction Program," NYSDOT. 2001. Hatcher, C. W. "Grooving Streets and Highways Can Help Stop Skid Crashes." Traffic Engineering. 1989

[23] Meier et.al, "Towards real-time middleware for vehicular ad hoc networks", LNCS, Springer, 2005.

[24] CARS- Context Aware Rate Selection for Vehicular Networks

[25] Guido Gehlen and Georgios Mavromatis, INVENT-VMTL "A Web Service based middleware for Mobile Vehicular Applications".

[26] Erik Weiss et.al., "MYCAREVENT- Vehicular communications gateway for Car Maintenance and Remote Diagnosis".

[27] Jose Santa and Antonio F. Gomez-Skarmeta, "Sharing context-aware road and safety information", IEEE Pervasive Computing, Volume 8 Issue 3, 2009, pages 58-65.

[28] Jose Santa et.al., "A Multiplatform OSGi based Architecture for Developing Road Vehicle Services", IEEE 2007, page 706-710. 


\section{Authors Information}

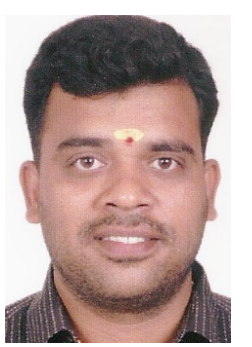

K. Saravanan is working as Assistant Professor Senior in School of Computer Science \& Engineering at VIT University. His research work focuses on Context-Aware Systems, Vehicular Ad hoc Networks, Middleware Technologies, Semantic Web and Web Services.

Email:saravanank@vit.ac.in .

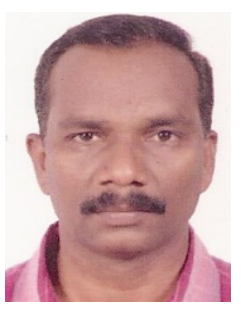

K Ramesh Babu is working as Associate professor in School of Computer Science \& Engineering at VIT University. His area of research work mainly focuses in the field of Quality of Service issues in adhoc networks. He had numerous funded research projects to his credit. He has published international conference and Journal papers on adhoc networks, QoS issues.

Email: ramgetkar@gmail.com

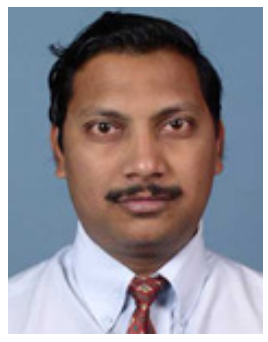

Arunkumar Thangavelu is currently associated with Vellore Institute of Technology University, India. He has over 16 years of academic, Research experience in field of adhoc, sensor networking, DataMining. $\mathrm{He}$ is an active consultant in R\&D planning, proposal evaluation as well reviewer in international / national journals. He had received his Doctoral Degree from PSG College of Technology. His domain of research expertise varies from mobile / vehicular adhoc networks, distributed computing to precision agriculture.

Email: arunkumar.thangavelu@gmail.com 